\title{
Biosystem for the culture and characterisation of epithelial cell tissues
}

\author{
S. Hediger ${ }^{\text {a }}$, J. Fontannaz $^{\text {a }}$, A. Sayah ${ }^{\text {a }}$, W. Hunziker ${ }^{\text {b }}$, M.A.M. Gijs ${ }^{\text {a }}$ \\ ${ }^{a}$ Institute of Microsystems, Swiss Federal Institute of Technology Lausanne, CH-1015 Lausanne EPFL, Switzerland \\ ${ }^{\mathrm{b}}$ Institute of Biochemistry, University of Lausanne, CH-1066 Epalinges UNIL, Switzerland
}

Received 1 July 1999; received in revised form 7 December 1999; accepted 7 December 1999

\begin{abstract}
We have designed and realised a new type of microsystem for the electrical characterisation of epithelial cell layers for biomedical diagnostic purposes. We have used deep plasma and other micromachining procedures for the fabrication of vias and microfluidic channels in silicon and glass substrates. Miniaturised cell culture devices have been realised by gluing nano-porous polycarbonate membranes in between two structured wafers and by combining these with glass wafers to provide integrated microfluidic channels. We have characterised the impedimetric properties of our microsystem, demonstrated epithelial cell layer growth within it, and have done the initial electrical characterisations of epithelial cell layers. We believe that our devices will open new perspectives for cell-based diagnostic applications, especially for those where available cell quantities pose a limiting factor. (C) 2000 Elsevier Science S.A. All rights reserved.
\end{abstract}

Keywords: Epithelial cell; Biological microsystem; Silicon; Micromachining; Cell culture; Microfluidics; Electrical resistance

\section{Introduction}

Miniaturised bio-chemical measurement systems will have a large impact on the way medical diagnostics will be done in the near future. More particularly, in the field of clinical diagnostics and pharmacology, the use of living cells for fast specific and non-specific chemical sensing is an area of increasing importance [1-3]. For example, cells in a liquid environment can be transported within microchannels by using electro-osmotic flow by applying high voltages across the microchannel [4,5]. A cell or cell layer is a complex system with appropriate response to a variety of external physical and chemical excitations. Experiments on such living biological systems (e.g. using electrical resistance or fluorescence measurements [6]) can lead to the study of diffusion and transport of biological or pharmacological molecules through the cell or cell layer [7,8]. Until now, all these experiments were done in macroscopic tissue resistance measurement chambers [9,10]. Individual cell culture chambers, with a diameter ranging from a few millimetres up to some centimetres, are realised in arrays in standard plate compatible formats. In these devices, a cell tissue layer is grown on a nano-porous

\footnotetext{
* Corresponding author. Tel.: +41-21-693-6585; fax: +41-21-6935950.

E-mail address: serge.hediger@epfl.ch (S. Hediger).
}

membrane placed at the bottom of the chamber and measured with two pairs of electrodes, which are mechanically inserted in the system after cell culture. Despite the functionality of these devices, there are several drawbacks. First of all, the relatively large size of the cell chamber requires relatively large amounts of cells and biological fluids. Also, the requirement of a perfectly closed cell culture layer (to prevent electrical short-circuiting events) is a condition, which is much harder to fulfil for the larger membrane surfaces. Moreover, the non-integrated and mechanically positioned electrodes are a source of uncertainty in determining the correct cell layer resistance, as varying contact geometries can give rise to large resistance contributions of the physiological cell medium. Evidently, the application of external electrical contacts also can be a source of cell culture contamination. The progresses in the field of microfabrication technology for the fabrication of three-dimensional microsystems have opened new ways in miniaturisation. Evidently, small millimetre-size or smaller cell culture chambers and membranes in a microsystem configuration with integrated electrical contacts and microfluidic feed-throughs do not suffer from all problems mentioned above.

In this paper, we propose a new microsystem approach, using miniaturised electrical measurement electrodes and integrated microfluidic channels, for the electrical characterisation of epithelial cell layers. The substrate chosen for 


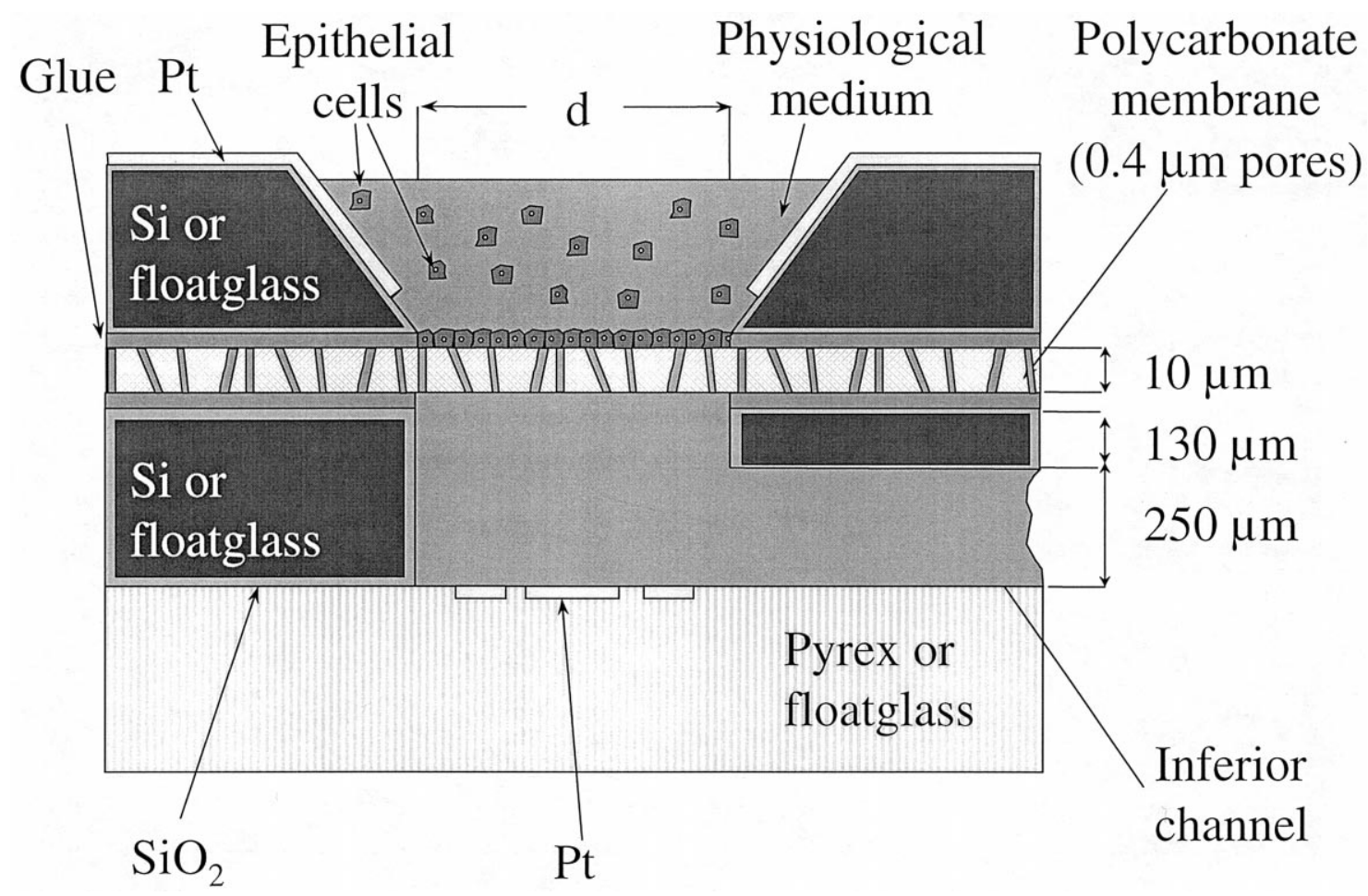

Fig. 1. (a) Schematic cross-section of the basic tissue measurement structure showing epithelial cell layer covered nano-porous membrane separating the top from the bottom reservoir.

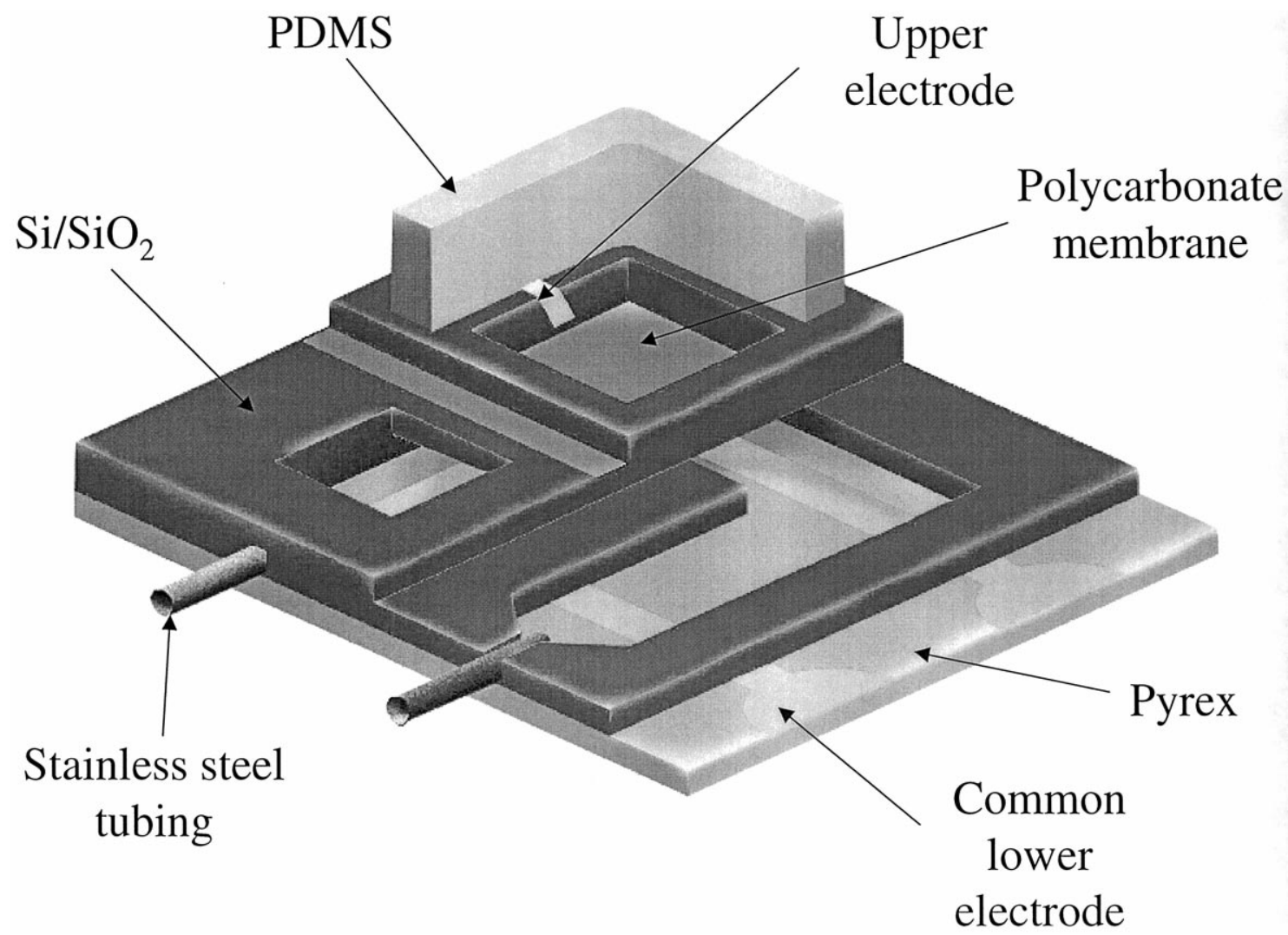

Fig. 2. Artist impression, showing an oblique view of a partially sectioned device revealing the various patterned layers as well as the top elastomer PDMS reservoir. 
the cell layer growth is a nano-porous polycarbonate membrane, as used in the macroscopic cell tissue measurement applications. This membrane is sandwiched between two micromachined wafers (silicon or glass) for providing upper and lower fluidic reservoirs, allowing cell nutrition and exposure to various chemicals from either side of the membrane. A lower Pyrex or floatglass wafer is combined with an intermediate wafer to form integrated microfluidic channels and connections to the membrane structures. Our structure facilitates control of the cell layer growth, the measurement of the cell layer resistance, the transport and diffusion of biological or pharmacological molecules through the cell layer and accurate measurement of (bio-) chemically induced resistance variations. We have realised this microsystem by using photolithography, various etching procedures (among which is silicon deep plasma etching and powder-blasting [11]), anodic wafer bonding and gluing technologies. We have characterised the electrical behaviour of our microsystem using $\mathrm{NaCl}$ and physio- logical liquid solutions and have done cell culture experiments on our devices. Finally, we have measured the frequency dependent surface resistance of Madin-Darby Canine Kidney (MDCK) cell layers grown on our devices.

\section{Design}

Fig. 1 shows a schematic cross-section of the basic tissue measurement structure: it consists of an assembly of two micromachined silicon or floatglass wafers, with a nano-porous polycarbonate membrane glued in between. The surface of a single culture device (determined by the free area of the membrane) ranges between 1 and $4 \mathrm{~mm}^{2}$. Before assembly, Ta/Pt electrodes are micropatterned on the upper silicon or floatglass wafer (forming the top reservoir) and on the lower Pyrex or floatglass wafer, respectively. The basic tissue measurement structure of Fig. 1 is repeated to form four separate and identical cell
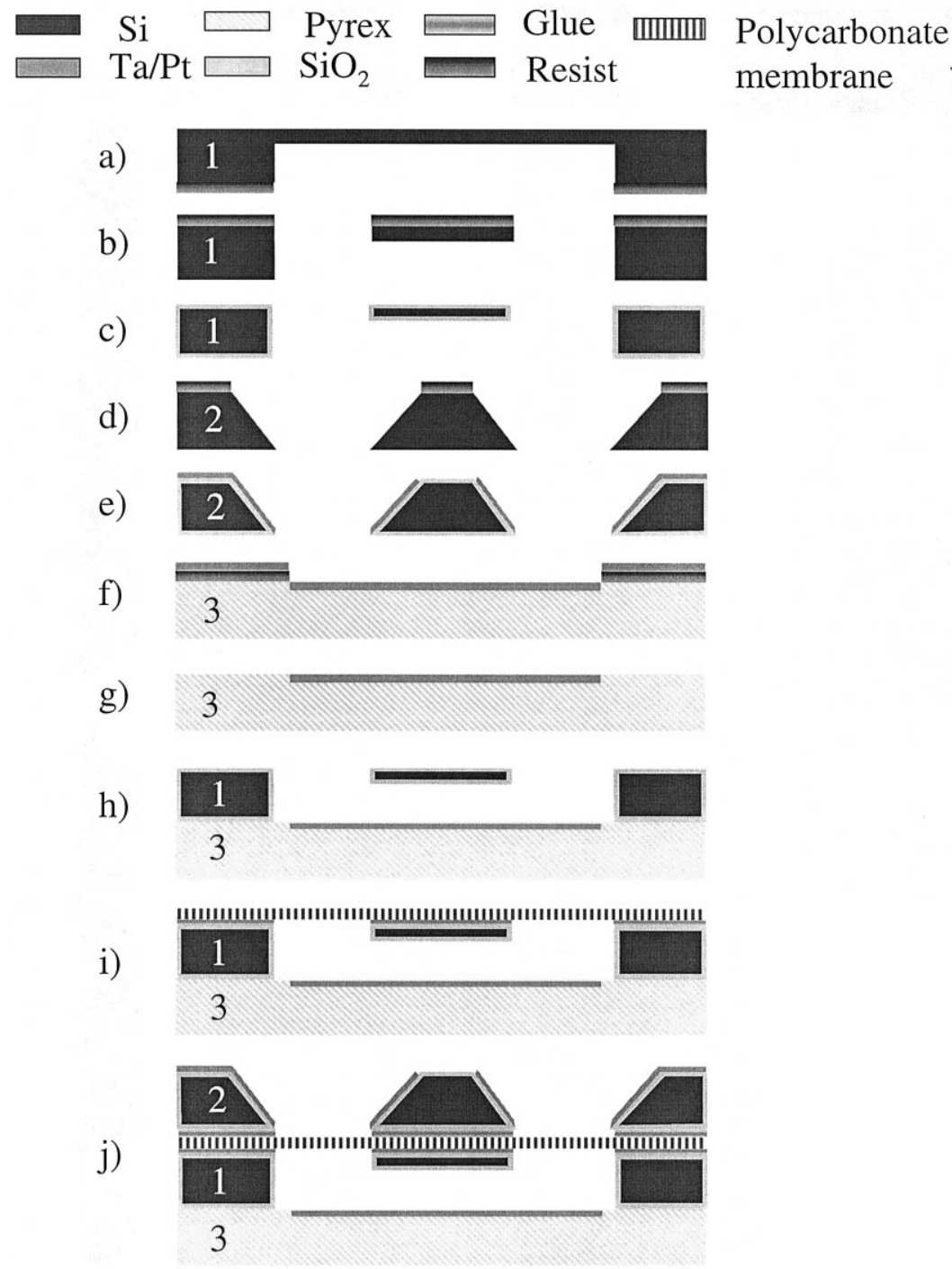

Fig. 3. Batch process for the realisation of the silicon based tissue culture measuring device, using two silicon wafers $(1,2)$ and one Pyrex wafer $(3)$. 
culture wells on one device. Fig. 2 is an artist impression, showing an oblique view of a partially sectioned device revealing the various patterned layers. This figure clearly shows the external fluidic connections to the lower reservoir underneath the membrane, as well as a top removable reservoir, made out of the elastomer Polydimethylsiloxane (PDMS), which is needed for providing a sufficiently large quantity of biological solution (withstanding evaporation) during the 3 days of the cell growth. The figure also shows the bottom electrodes in the lower microfluidic channel and the upper electrodes on the top wafer.

\section{Microfabrication technology}

Fig. 3 shows the batch process for the realisation of the silicon based tissue culture measuring device. Five lithographic masks are used for the whole processing sequence. The fabrication technologies are deep plasma etching for the cell culture via and for the microfluidic channel in the lower silicon wafer, wet etching for the top silicon well, anodic bonding of a Pyrex glass wafer with the lower silicon wafer and, finally, an appropriate gluing procedure for sandwiching the polycarbonate membrane in between the two silicon wafers.

We start with a 380- $\mu$ m thick 4" (100) silicon wafer with a resistivity of $0.3 \Omega \mathrm{cm}$, on which we structure a photoresist mask for the dry etching of the vias and microfluidic channels in the lower silicon wafer (denoted by 1 in Fig. 3). We used a $\mathrm{SF}_{6}$ deep plasma etch, combined with a $\mathrm{C}_{4} \mathrm{~F}_{8}$ sidewall passivation process in a STS reactor, resulting in vertical etch profiles and etch rates of about 5 $\mu \mathrm{m} / \mathrm{min}$ (Fig. 3a,b). The same kind of silicon wafer (denoted by 2 in Fig. 3) is used as top substrate for the etching of the wells in a classical $\mathrm{KOH}$ solution in order to obtain oblique sidewalls (Fig. 3d). After a thermal oxidation step giving rise to an oxide thickness of about $0.5 \mu \mathrm{m}$ on every outer silicon surface, including the via, well and

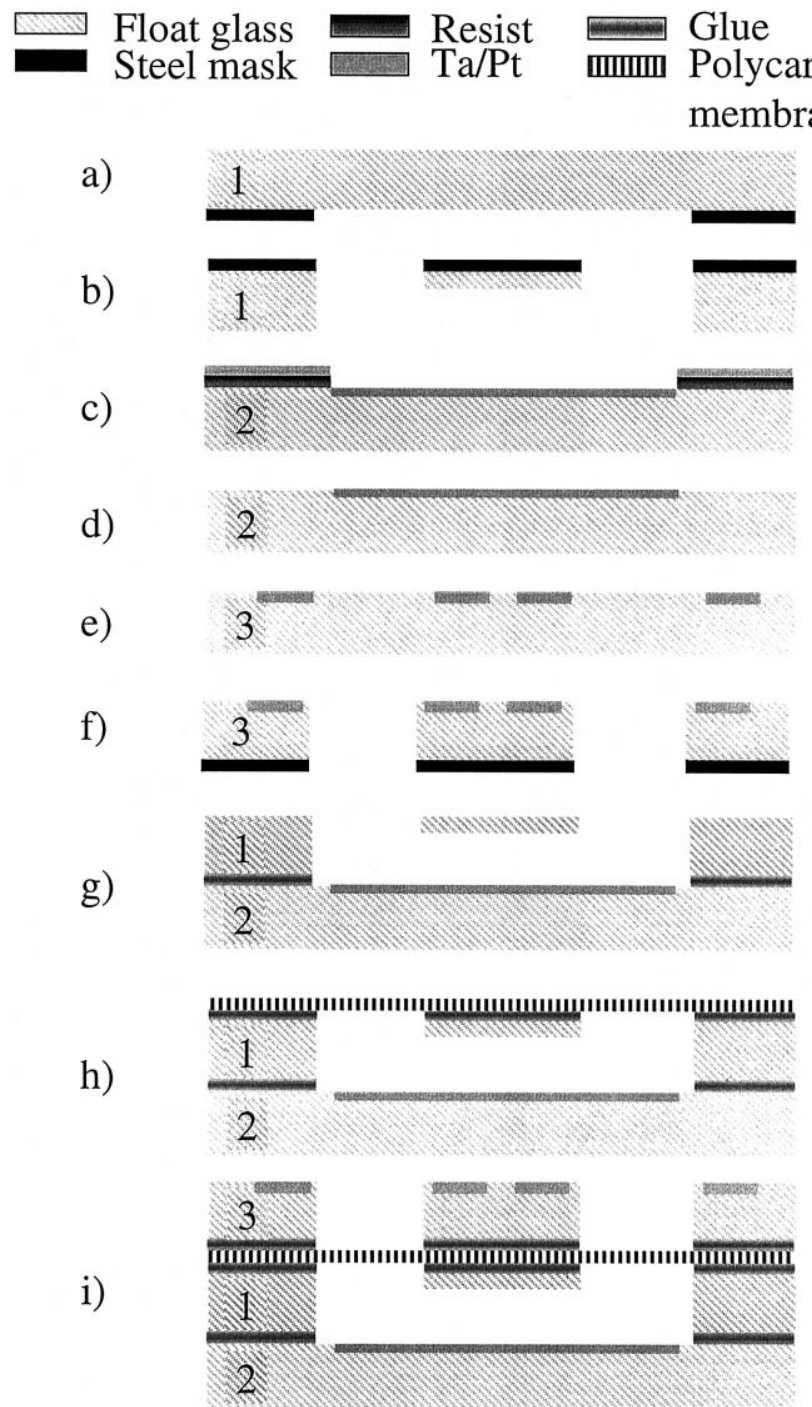

Fig. 4. Batch process for the realisation of the glass based tissue culture measuring device using floatglass substrates. 
the microfluidic channel (Fig. 3c), the top Ta/Pt electrodes are sputtered through a shadow mask. The electrical contacts on the Pyrex wafer (denoted by 3 in Fig. 3) are realised using a lift-off process (Fig. 3f,g). However, prior to the Pt deposition, we use the lift-off stencil to etch a $0.5-\mu \mathrm{m}$ deep recess in which the $\mathrm{Ta} / \mathrm{Pt}$ contacts will be buried (Fig. 3f). This procedure gives rise to a flat top wafer surface, which lends itself excellently to an anodic bonding assembly with the top silicon wafer (Fig. 3h). Fig. $3 \mathrm{i}$ represents the gluing of the nano-porous membrane on the structure of Fig. 3h. The structure is completed by gluing the structured silicon wafer of Fig. $3 \mathrm{~d}$ on top of the membrane, forming the upper reservoirs.

Fig. 4 shows the batch process for the realisation of the glass-based tissue culture measuring device. The sequence is similar to the one used for the silicon-based device. The plasma and $\mathrm{KOH}$ etching procedures are replaced by a powder-blasting erosion process using metallic contact masks. The various structured layers are bonded simply by gluing.

We start with a 530- $\mu \mathrm{m}$ thick $3^{\prime \prime}$ floatglass wafer (denoted by 1), on which we pattern a channel and inlet and outlet vias using powder-blasting through a metal contact mask (Fig. 4a,b). The top and bottom $\mathrm{Ta} / \mathrm{Pt}$ electrodes are then realised by sputtering on two other floatglass wafers (denoted by 2 and 3) using a lift-off process (Fig. 4c,d and e). The glass wafer of Fig. 4e (which will be the wafer, containing the top electrical contacts of our device) is powder-blasted through a metallic mask, and will later form the top cell culture chambers (Fig. 4f). The same gluing technology as for the siliconbased device is then used to glue the bottom wafer with the glass wafer containing the channels of Fig. 4d (Fig. $4 \mathrm{~g}$ ). The nano-porous membrane and the top glass wafer are as well glued with the same gluing technology to obtain the finalised device (Fig. 4h,i).

One of the most critical steps for the fabrication of the tissue culture measurement device is the polycarbonate membrane gluing. The whole difficulty lies in the fact that the polycarbonate membrane has to remain free of glue on each of the cell culturing sites. The method used is schematically shown in Fig. 5. It consists of applying an ultra-thin layer of liquid glue (Araldit ${ }^{\circledR}$ ) on a paraffin foil using a rolling procedure and bringing it into contact with the lower stack of the device (Fig. 5a). By carefully lifting the paraffin foil, a smooth an thin $(\sim 1 \mu \mathrm{m})$ gluing layer is
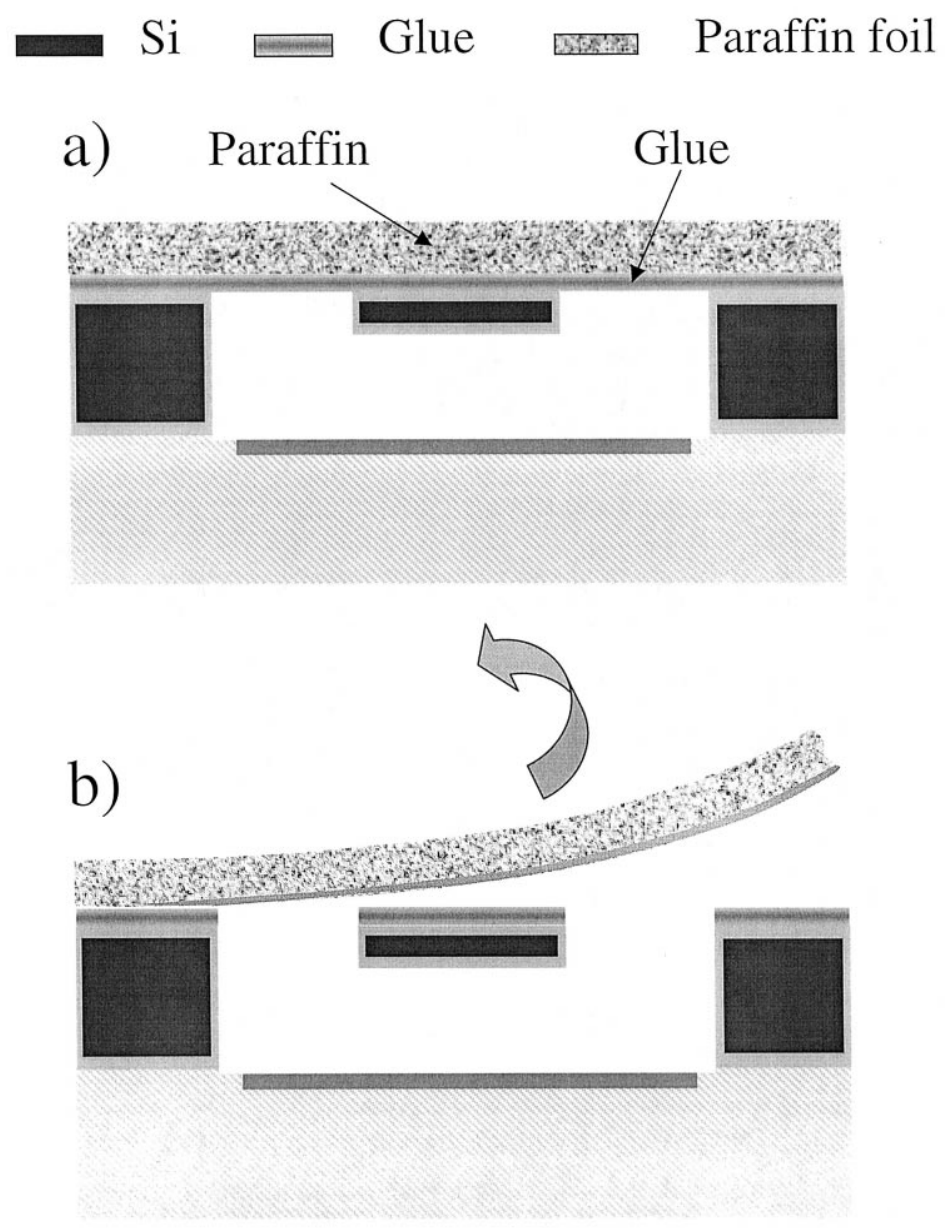

Fig. 5. Schematic of the gluing procedure chosen to obtain a membrane free of glue. 


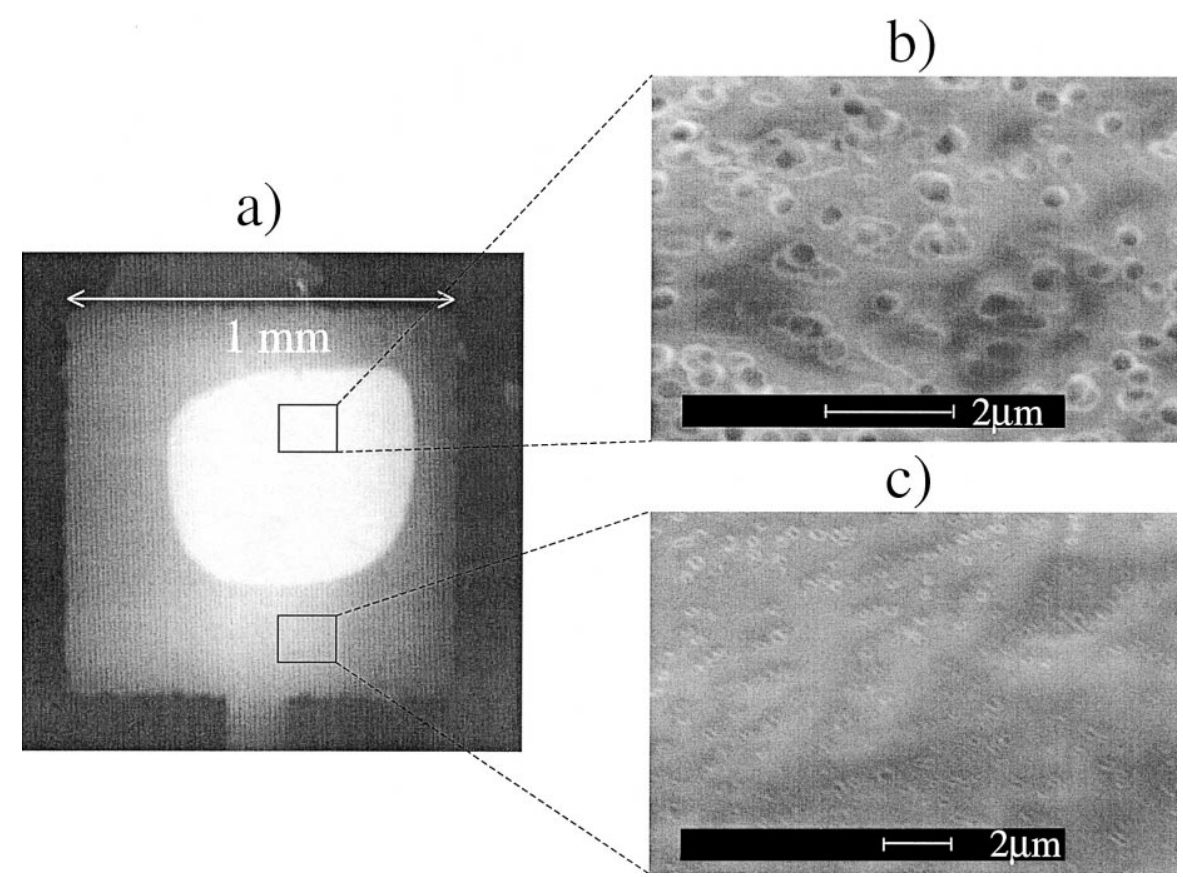

Fig. 6. Optical and SEM photographs of glued polycarbonate membranes: (a) membrane partially covered glue due to unoptimised gluing method; (b) part of the nano-porous membrane free of glue; (c) part of the nano-porous membrane covered with glue.

left on the surface (Fig. 5b). A similar procedure is adopted for applying glue on the lower side of the top wafer. The gluing procedure appeared to be very critical in the fabrication process; when the gluing layer is not suffi-

(a)

\section{$\mathrm{KOH}$ etched top reservoir}

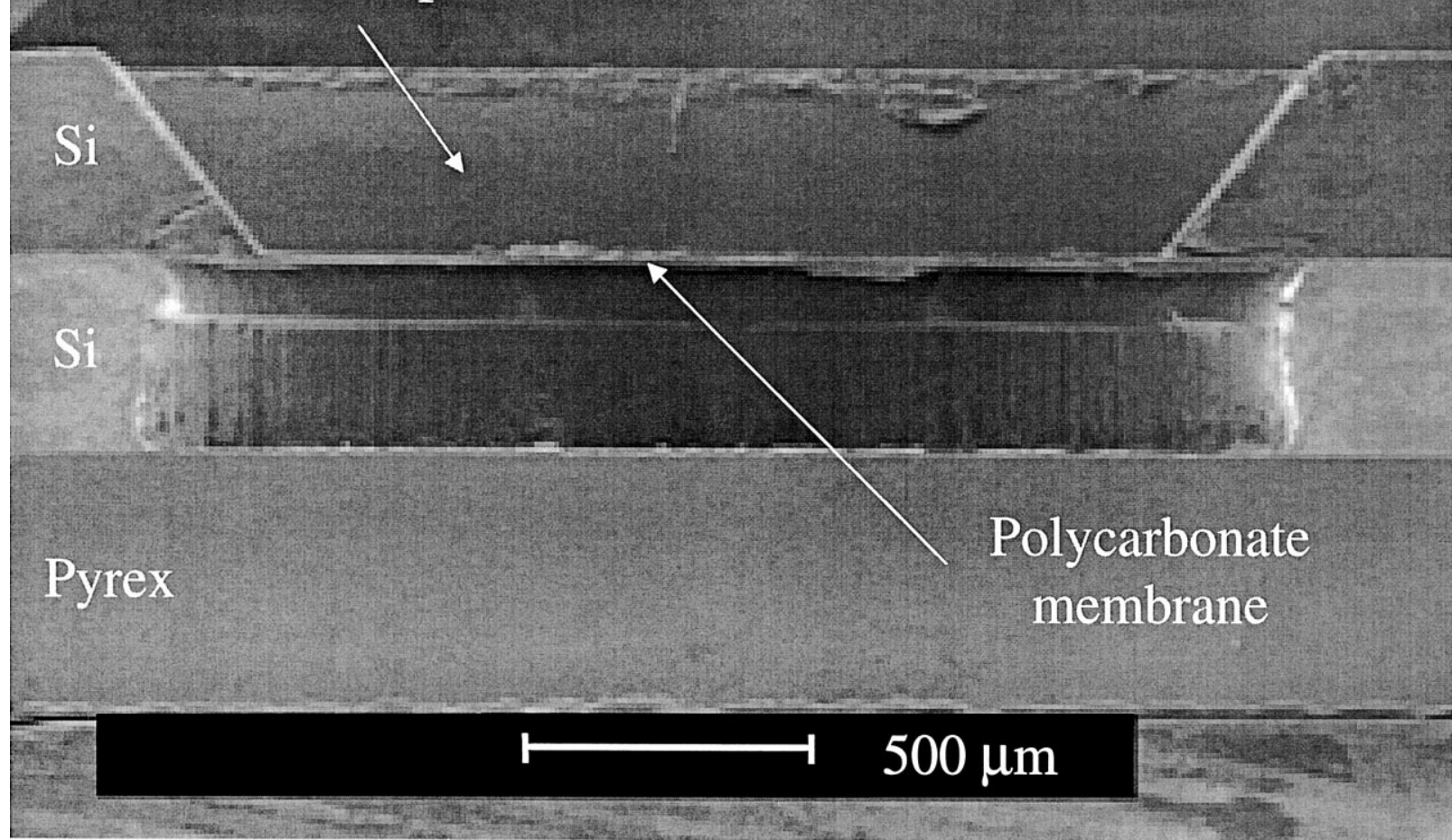


(b)

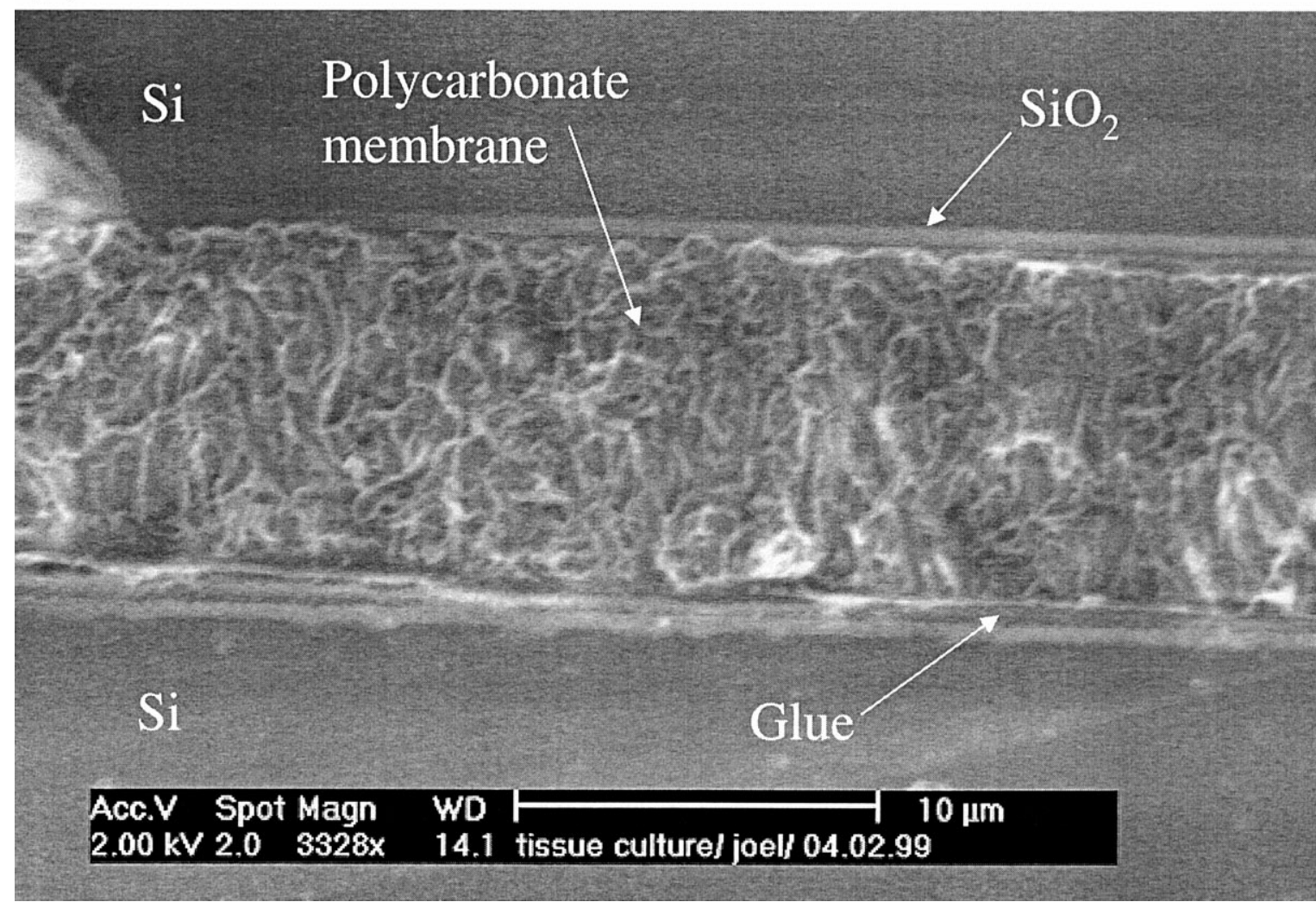

Fig. 7. (a) SEM cross-section of the complete stack of a cell culture device showing the nano-porous membrane sandwiched between two silicon wafers and forming separate top and bottom reservoir. (b) Detailed SEM cross-section of the glued polycarbonate nano-porous membrane.

ciently thin, small residues of glue tend to spread on the polycarbonate membrane, thereby filling the nano-pores and preventing later cell culture at such site. Evidently, when the nano-porous membrane surface is partially covered with glue, this will result in an arbitrary and variable enhancement of the measured perpendicular resistance. This would be an additional origin in a spread of resistance values of grown cell layers during later device applications. For experiments with grown cell layers, we have used only devices with a nano-porous membrane fixed using our special gluing procedure and, hence, completely free of residual glue on the membrane.

\section{Results and discussion}

The gluing problem is illustrated in Fig. 6a, which shows a glued nano-porous membrane, of which an important part of the surface is covered with glue. For this sample, we did not use our special gluing procedure described above. The device is characterised by a much smaller cell culture area. Scanning Electron Microscope (SEM) graphs of both uncovered and glue covered membrane surface are shown in Fig. $6 \mathrm{~b}$ and c, respectively. Fig. $7 \mathrm{a}$ and $\mathrm{b}$ are SEM cross-sections of the complete stack of a silicon based cell culture device, Fig. 7b showing a detail of the membrane with its two gluing layers. Fig. 8a and b are photographs of diced and Printed Circuit Board (PCB) mounted device, Fig. 8a showing the silicon based device with electrical connectors on the left and microfluidic connectors on the right of the structure. Fig. $8 \mathrm{~b}$ shows the glass-based device with the PDMS reservoir attached onto the top silicon wafer, allowing the application of sufficient physiological fluid during the growth of the epithelial layer.

After the design, the technology development and the fabrication of our new microsystem for epithelial cell culture applications, we have characterised the frequency dependent electrical impedance $(100 \mathrm{~Hz}<f<2 \mathrm{MHz})$ of our devices, using a HP4194A Impedance/Gain-Phase analyser. Therefore, we have used $\mathrm{NaCl} 0.2 \mathrm{M}$ salt solutions $(\rho \approx 60 \Omega \mathrm{cm})$ and physiological solution $(\rho \approx 60$ $\Omega \mathrm{cm}$ ) used during growth of the epithelial cell layers. Fig. 9a shows the impedance of a silicon- and a glass-based cell culture device, filled with physiological solution, as a function of frequency. At low frequencies, the impedance is particularly high; we attribute this to the large difference in resistivity between the physiological fluids and the metal electrodes, giving rise to a polarisation layer near the electrodes, resulting in an effective capacitance, as is well 


\section{a)}

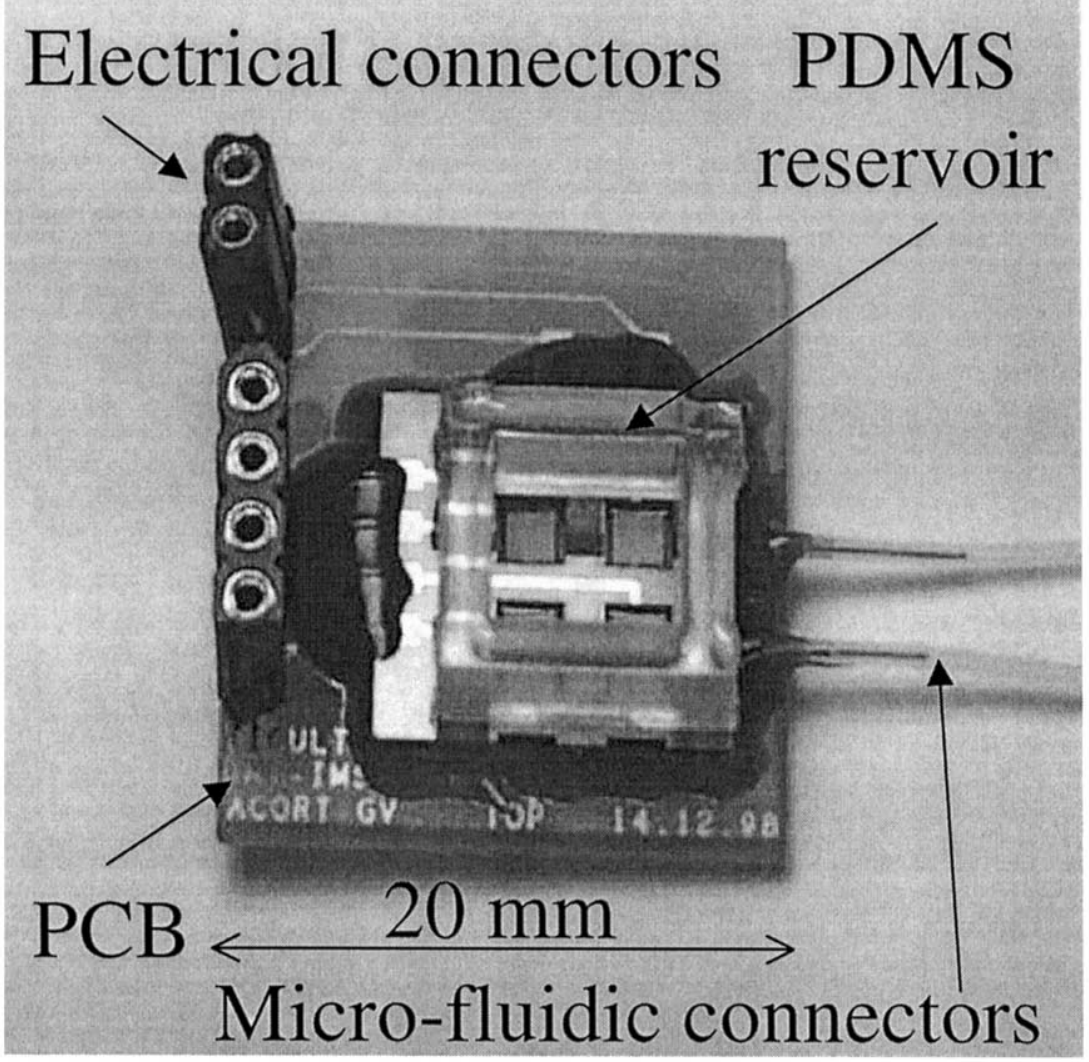

b)

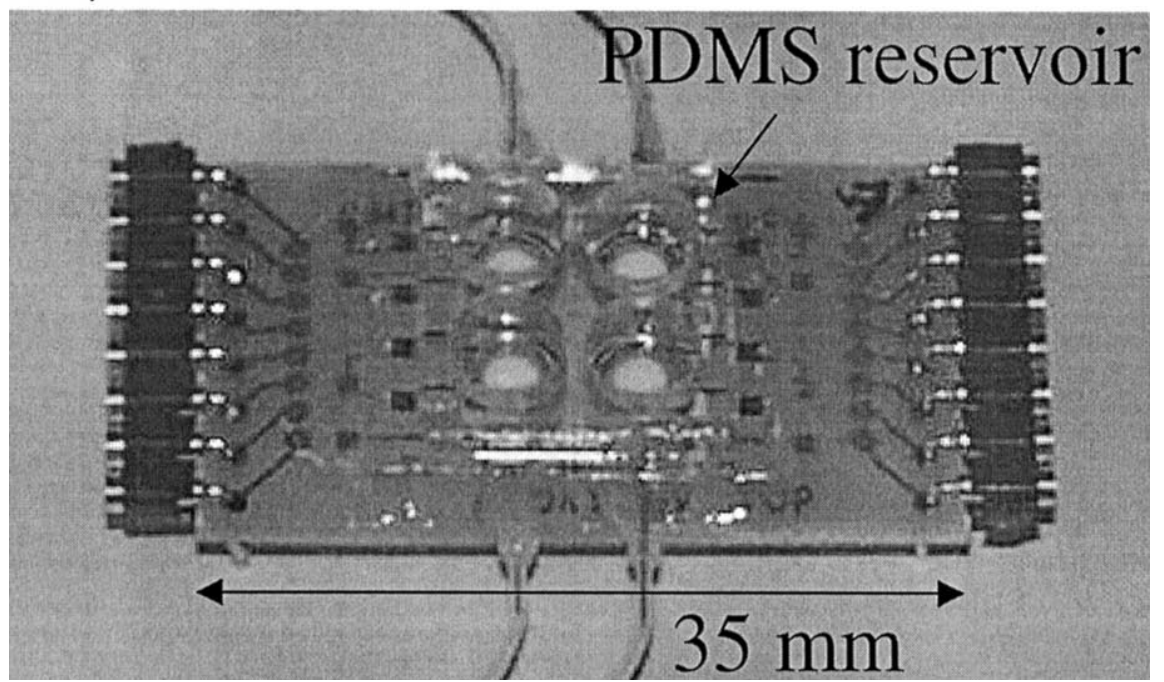

Fig. 8. Optical photographs of diced and PCB mounted devices: (a) silicon-based device (b) glass-based device.

known for a Schottky diode [12]. With increasing frequency, this electrode capacitance becomes short-circuited, resulting in a lower impedance corresponding with the actual resistance of the nano-porous membrane and the physiological liquid within the channels and vias. For example at $100 \mathrm{kHz}$, we measure a surface impedance of 25 and $30 \Omega \cdot \mathrm{cm}^{2}$ for the silicon- and the glass-based device, respectively. For a better understanding of our 

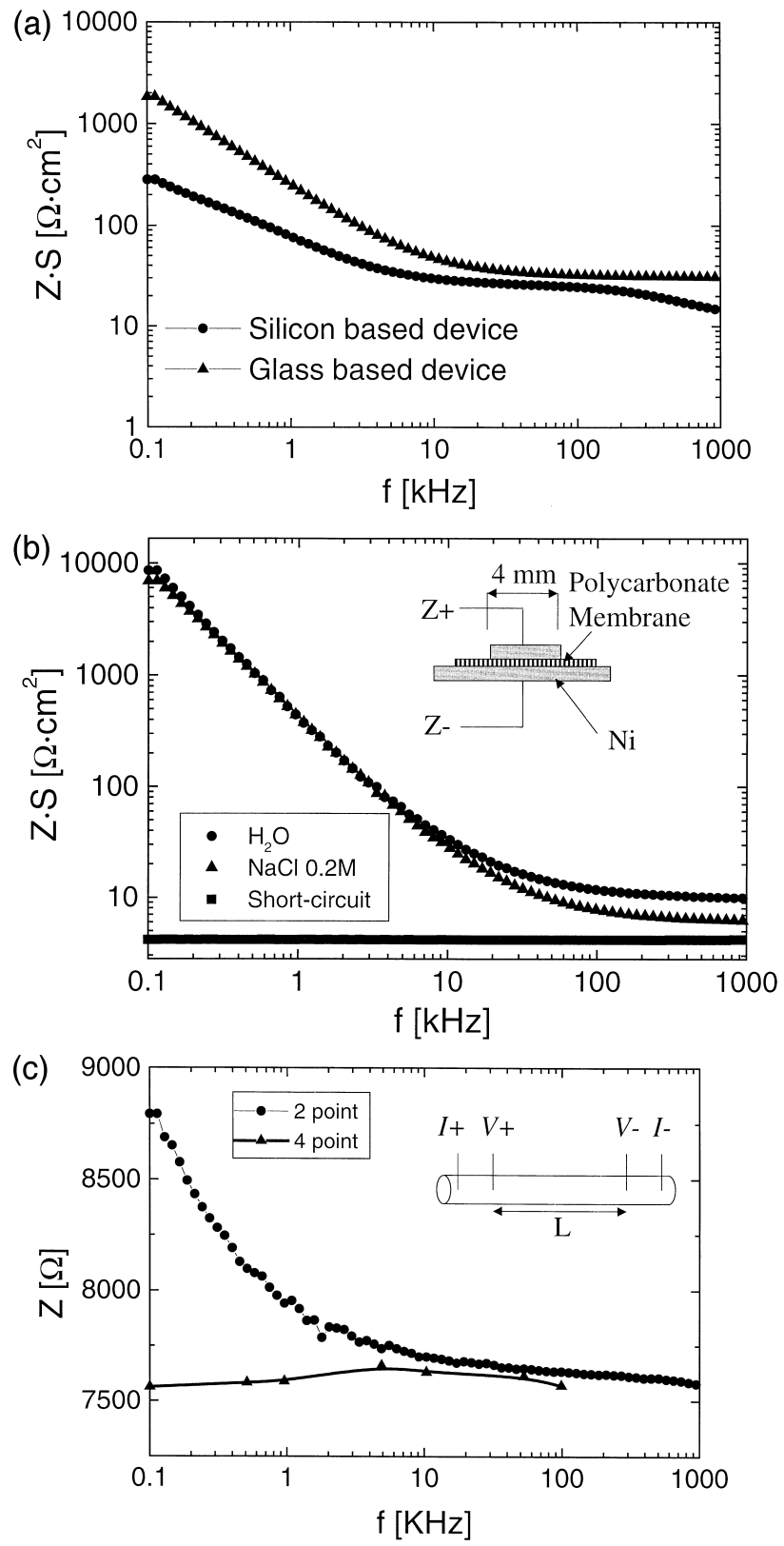

Fig. 9. (a) Frequency dependent two-point surface impedance measurement in both types of biosystem using physiological solution $(\rho=60$ $\Omega \mathrm{cm}$ ). (b) Frequency-dependent two-point impedance measurement of a polycarbonate membrane with $0.4 \mu \mathrm{m}$ pores and a surface area $S=16$ $\mathrm{mm}^{2}$. We used two flat $\mathrm{Ni}$ electrodes to have the electrical field perpendicular to the membrane and distilled water and $\mathrm{NaCl} 0.2 \mathrm{M}$ solution $(\rho=60 \Omega \mathrm{cm})$ as pores filling solution. (c) Frequency dependent twoand four-point impedance measurements in a plastic tube of 1.4-mm diameter and 20-mm length using a physiological solution $(\rho=60$ $\Omega \mathrm{cm}$ ).

results, we have realised in parallel a few impedance reference measurements on another type of structure, in which the electrical field is perfectly perpendicular to the membrane, shown in Fig. 9b. We have sandwiched a same type of nano-porous membrane filled with a $\mathrm{NaCl} 0.2 \mathrm{M}$ solution between two metal $(\mathrm{Ni})$ electrodes, thereby avoid- ing direct electrical contact via the liquid from the top $(\mathrm{Z}+)$ to the bottom $(\mathrm{Z}-)$ electrode. The surface of the upper electrode, defining the resistance measurement area, is $16 \mathrm{~mm}^{2}$. The lower curve of Fig. $9 \mathrm{~b}$ shows the measured impedance when no polycarbonate is used and the upper electrode is brought into contact with the bottom one without use of solution. For this situation, we find a resistance of $4 \Omega \cdot \mathrm{cm}^{2}$ for our two point electrode measurement set-up, which should be subtracted from the experimental curves. The resistance results are very similar to that of Fig. 9a. For example, for the $\mathrm{NaCl}$ solution, we find a resistance per unit surface area of $7.5 \Omega \cdot \mathrm{cm}^{2}$ at 100 $\mathrm{kHz}$, corresponding to the true perpendicular resistance of the membrane and consistent with the electrical measurement of Fig. 9a.

To further elucidate the influence of electrode configuration on the electrical measurements, we also have verified our hypothesis of the Schottky barrier-like induced capacitance near the metal-liquid interface by resistance control experiments on $\varnothing 1.4 \mathrm{~mm}$ plastic tubes filled with $\mathrm{NaCl}$ and physiological solution and provided with four metallic contacts. The result of a two point measurement of the liquid column impedance is shown as the upper curve of Fig. 9c. One clearly observes the impedance rise at low frequencies, similar to Fig. 9a and b. On the other hand, for the four point measurement, we observe a nearly flat curve, which corresponds to the proper frequency independent impedance and resistivity of the liquid. This experiment indicates that valuable information on the membrane and cell layer impedance can be obtained by measuring at high frequencies, even in the two-point set-up.

Fig. 10 is a photograph of an epithelial cell monolayer grown on our microstructure. MDCK cells possess characteristics similar to the principal cells of the collecting kidney duct and form tight epithelial-like monolayers when grown on permeable supports. Cells (originally obtained

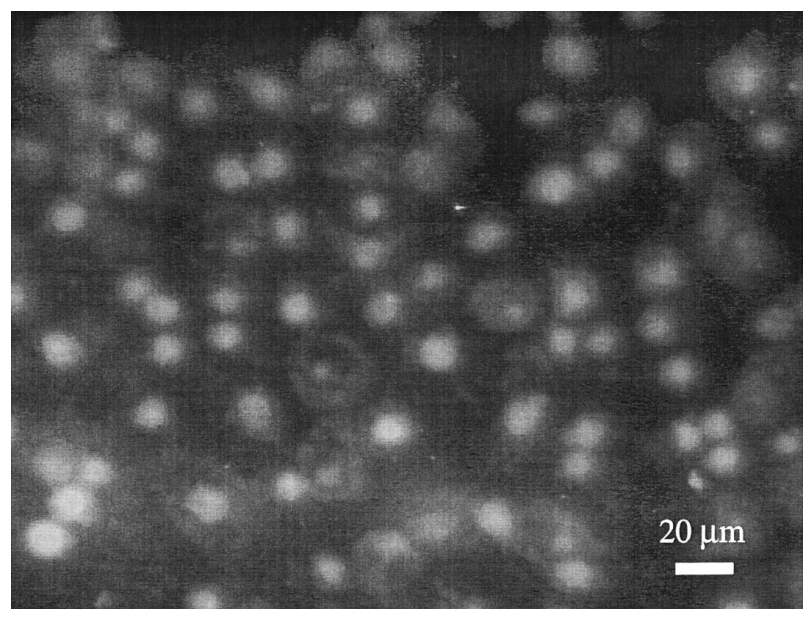

Fig. 10. Optical photograph of fluorescently labelled cell nuclei of an epithelial cell layer grown into our microsystem (MDCK cells are used). 


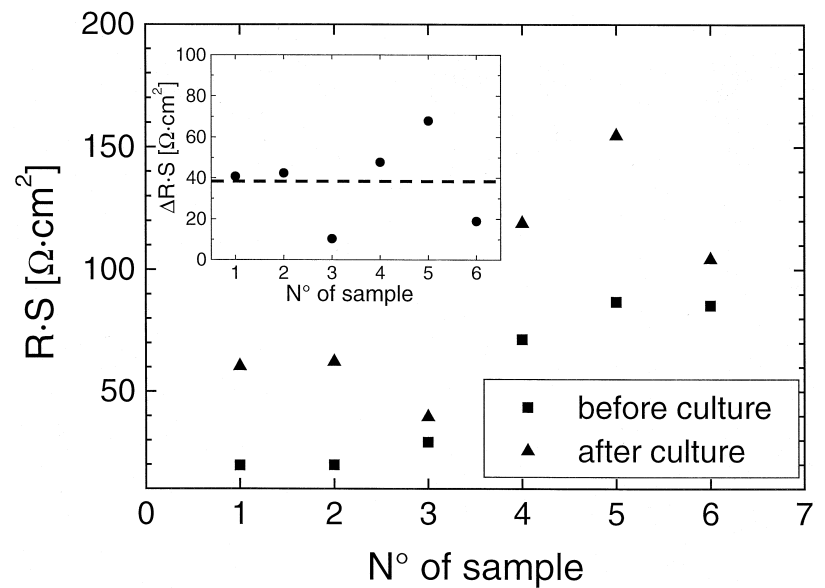

Fig. 11. Four point surface resistance measurements at $1 \mathrm{kHz}$ in six nominally identical culture sites of our glass-based microsystems, before and after epithelial cell culture. The insert shows the difference of surface resistance before and after cell culture. The dashed line represents the mean value.

from K. Matlin, Boston) were cultured as described [13] and plated on the microstructures at a density of $5 \times$ $10^{4} / \mathrm{cm}^{2}$ for 3 days to obtain a confluent monolayer. The cells on the microstructures where then fixed in ethanol/acetic acid $\left(3: 1 ; 5 \mathrm{~min}\right.$ at $\left.-20^{\circ} \mathrm{C}\right)$, washed in $\mathrm{H}_{2} \mathrm{O}$ (5 min, room temperature) and the nuclei stained with the Hoechst 33258 bisbenziamid fluorochrome (50 $\mathrm{ng} / \mathrm{ml}$ in $\mathrm{H}_{2} \mathrm{O}$ for $30 \mathrm{~min}$ at $37^{\circ} \mathrm{C}$ ). After washing in $\mathrm{H}_{2} \mathrm{O}$ (5 min at room temperature), the cells on the microstructure were covered with a drop of Moviol embedding solution [10] and viewed with a Zeiss Axiophot microscope using a $63 \times$ Apachromat oil immersion lens, epifluorescence and fluorescein filters. The cell-growth experiments demonstrate the potential of our microsystem for miniaturised biological applications.

Fig. 11 shows the surface resistance measurement in our glass-based devices before and after an MDCK cell culture. Four point surface resistance measurements were performed at $1 \mathrm{kHz}$ in six nominally identical culture sites of our glass-based microsystems. The insert shows the difference of surface resistance before and after cell culture. The dashed line represents the mean value. The mean difference of surface resistance value is of about $40 \Omega \cdot \mathrm{cm}^{2}$ with a considerable standard deviation. As we have used only devices with a nano-porous membrane fixed using our special gluing procedure and, hence, completely free of residual glue on the membrane, this resistance variation is not due to the gluing procedure but due to the non-uniformity of current lines in our device, combined with slight geometrical differences between the culture sites. Indeed, when locating the measurement electrodes very close to the membrane, the measurement will become very sensitive (as practically, the resistance of the fluid does not contribute to the measured resistance), but the exact mea- sured value will strongly depend on contact symmetry, positioning with respect to the cell chamber, etc. The mean value for the surface resistance is of the same order of magnitude as the results on the 'macroscopic' epithelial cell culture devices, where we found a value of $70 \Omega$. $\mathrm{cm}^{2}$. The difference in surface resistance between the macroscopic cell culture devices and our microsystem approach could be due to edge effects of the membrane during cell culture or due to non-uniform measuring currents (for the 'macroscopic' devices).

\section{Conclusions}

We have designed and realised a new type of microsystem for the electrical characterisation of epithelial cell layers for biomedical diagnostic purposes. We have used several fabrication technologies such as deep plasma, wet etching procedure and powder-blasting, as well as metal thin film deposition in order to obtain microfluidic channels in silicon or glass substrates, with patterned electrodes. The miniaturised cell culture device was then assembled using a well-studied gluing procedure, whereby we sandwich a nano-porous polycarbonate membrane between two structured wafers. We have used only devices with a nano-porous membrane fixed using our special gluing procedure and, hence, completely free of residual glue on the membrane, thereby avoiding device related perpendicular resistance variations. Devices have been systematically tested using electro-physiological fluid and ionic solutions of various resistivity. We have characterised the electrical behaviour of our microsystems using frequency-dependent impedance measurements. Finally, we have demonstrated epithelial cell layer growth within our microsystem and surface resistance measurements of the cell mono-layer. We hope that our devices will open new perspectives for cell based diagnostic applications, especially for those where available cell quantity is a limiting factor.

\section{References}

[1] G. Fuhr, Examples of three-dimensional microstructures for handling and investigation of adherently growing cells and submicron particles, Anal. Methods Instrum. (1996) 39-54, Special Issue $\mu$ TAS '96.

[2] G. Fuhr, S.G. Shirley, Cell handling and characterisation using micron and submicron electrode arrays, J. Micromech. Microeng. 5 (1995) 77-85.

[3] N.B. Standen, P.T.A. Gray, M.J. Whitaker (Eds.), Microelectrode Techniques, The Company of Biologists, Cambridge, 1987.

[4] D.J. Harrison, P. Li, T. Tang, W. Lee, Manipulation of biological cells and of DNA on-chip, Anal. Methods Instrum. (1996) 147-149, Special Issue $\mu$ TAS ' 96 . 
[5] P.E. Andersson, P.C.H. Li, R. Smith, R.J. Szarka, D.J. Harrison, Biological cell assays on an electrokinetic microchip, Proc. Transducers '97, 1997 Int. Conf. On Solid-State Sens. Actuators, Chicago (1997) 1311-1314.

[6] S. Hediger, A. Sayah, M.A.M. Gijs, Fabrication of a novel microsystem for the electrical characterisation of cell arrays, Sensors and Actuators, B 56 (1999) 175-180.

[7] D.S. Misfeldt, S.T. Hammamoto, D.R. Pitelka, Transepithelial transport in cell culture, Proc. Natl. Acad. Sci., USA 73 (1976) 12121216.

[8] L.C. Milks, M.J. Brontoli, E.B. Cramer, Epithelial permeability and the transepithelial migration of human neutrophils, J. Cell Biol. 96 (1983) 1241-1247.

[9] Corning Costar ${ }^{\circledR}$ product literature, Corning Costar, Acton, MA, USA, 1999.

[10] Falcon ${ }^{\circledR}$ product literature, Becton Dickinson, Franklin Lakes, NJ, USA, 1998.

[11] E. Belloy, S. Thurre, E. Walckiers, A. Sayah, M.A.M Gijs, The introduction of powder blasting for sensor and microsystem applications, Sensors and Actuators, A (1999) submitted.

[12] S.M. Sze, Physics of Semiconductor Devices, Wiley, Singapore, 1981.

[13] W. Hunziker, I. Mellman, Expression of macrophage-lymphocyte Fc receptors in Madin-Darby canine kidney cells: polarity and transcytosis differ for isoforms with or without coated pit localisation domains, J. Cell Biol. 109 (1989) 3291-3302.

\section{Biographies}

Serge Hediger was born in Vevey, Switzerland, in 1973. He received his degree in Microengineering from the Swiss Institute of Technology, EPF Lausanne, after a diploma project carried out in the Ceramics Institute in the field of piezoelectric accelerometers in March 1997. He joined the Institute of Microsystems in April 1998 as research assistant for new microsystems technologies for biomedical applications, in particular, for living cell electrical measurement devices.

Joel Fontannaz was born in Fully, Switzerland. He received his degree in Microengineering in 1999 from de Swiss Federal Institute of Technology, ETH Lausanne. He carried out his diploma thesis in the Institute of Microsystems, Group Technology, where he has designed and technologically realised a micromachined structure for the electrical characterisation of living cells for diagnostic biomedical purposes.
Abdeljalil Sayah received his degree in Physics in 1988 from University Mohammed V, Rabat, specialising in state solid physics. He obtained his AESA and DEA in Microelectronics specialising in the technology of the components in 1990 and 1992, respectively. From 1993 until 1996, he worked as a research assistant in the CNET-Bagneux (Paris) Laboratory where he received a $\mathrm{PhD}$ degree for the thesis entitled "Realisation of Silicon-based Dielectrics Optical Waveguide on InP by Photochemical Deposition'. In 1996, he joined the Applied Optics Institute (IOA) of the Swiss Federal Institute of Technology in Lausanne (EPFL) as a post-doctoral research. His research interests included the study and characterisation of optical fiber tips for scanning near field optical microscopy by protection layer liquid-phase etching and participated on the characterisation of Bragg grating produced in optical fibers by UV laser. He is now with the Institute for Microsystems (IMS) at EPFL. His current research interests are in inertial sensor (gyroscope), bio-sensors, and fabrication technologies.

Walter Hunziker received his degree in Natural Sciences from the Swiss Federal Institute of Technology Zurich (Switzerland) in 1983 and a PhD in Natural Sciences from the same university in 1886. Subsequently, he carried out studies on membrane traffic in epithelial cells at the Department of Cell Biology, Yale University School of Medicine, New Haven, CT. He joined the Institute for Biochemistry of the University of Lausanne (Switzerland) in 1992 as a group leader and professor. His research focuses on intracellular traffic of IgG Fc receptors in epithelial cells and the generation and maintenance of epithelial cell polarity.

Martin A.M. Gijs received his degree in Physics in 1981 from the Katholieke Universiteit Leuven, Belgium and his PhD degree in Physics at the same university in 1986. He joined the Philips Research Laboratories in Eindhoven, The Netherlands, in 1987. Subsequently, he has worked there on micro- and nano-fabrication processes of high critical temperature superconducting Josephson and tunnel junctions, the microfabrication of microstructures in magnetic multilayers showing the giant magnetoresistance effect, the design and realisation of miniaturised motors for hard disk applications and the design and realisation of planar transformers for miniaturised power applications. He joined the Swiss Federal Institute of Technology Lausanne (Ecole Polytechnique Fédérale de Lausanne) in 1997 as a professor in the Institute of Microsystems of the Microengineering Department, where he is responsible for the Microsystems Technology group. His main interests are in developing technologies for novel inductive-type devices, new microfabrication technologies for microsystems fabrication in general and the development and use of microsystems technologies for biomedical applications in particular. 